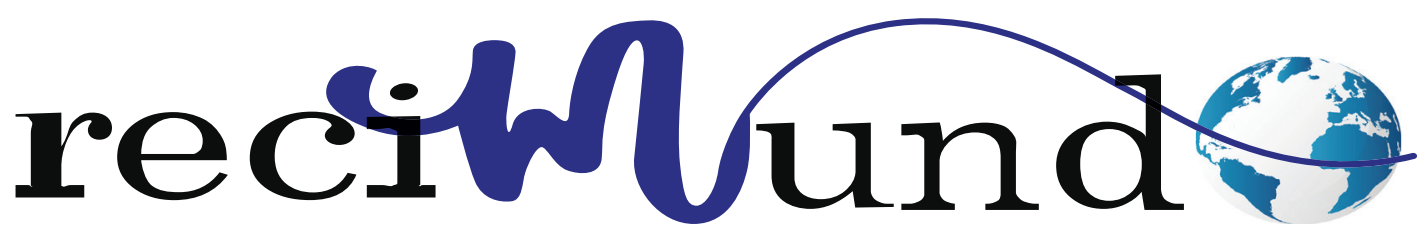

Revista Científica Mundo de la Investigación y el Conocimiento

DOI: 10.26820/recimundo/5.(2).julio.2021.101-107

URL: https://recimundo.com/index.php/es/article/view/1234

EDITORIAL: Saberes del Conocimiento

REVISTA: RECIMUNDO

ISSN: 2588-073X

TIPO DE INVESTIGACIÓN: Artículo de revisión

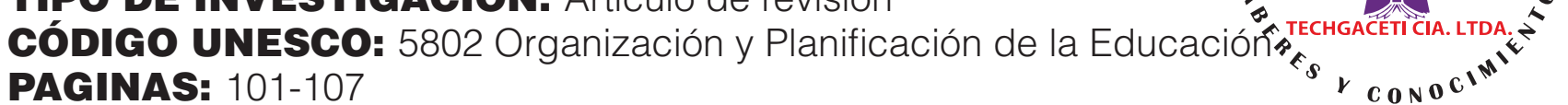

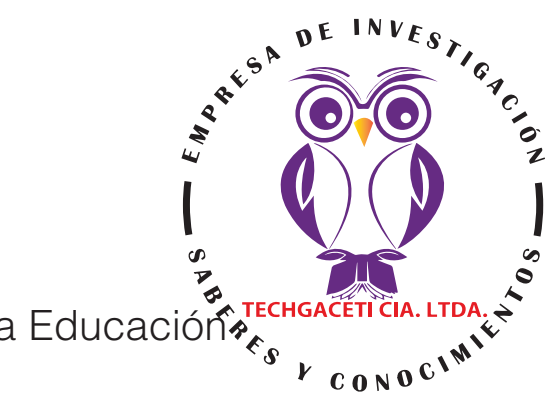

\title{
El rol del docente en la educación virtual
}

The role of the teacher in virtual education

0 papel do professor na educação virtual

Edelmira Lila Guevara Î̃ĩguez; Ruth Cecibelt Cedeño Álvarez²; Mario Enrique Escobar Gortaire3; Segundo Rafael Medina Velasco ${ }^{4}$

\section{RECIBIDO: 11/04/2021 ACEPTADO: 15/06/2021 PUBLICADO: 30/07/2021}

1. Magister en Informática Educativa y Multimedios mención Informática Educativa; Diploma Superior en Evaluación de la Calidad de las Instituciones de Educación Superior; Diploma Superior en Diseño de Proyectos; Diploma Superior en Comercio Electrónico; Doctora en Ciencias de la Educación mención Informática Educativa; Licenciada en Ciencias de la Educación mención Informática Educativa; Universidad Estatal de Bolívar; Ecuador; eguevara@ueb.edu.ec; (iD https:// orcid.org/0000-0002-2995-5885

2. Master en Pedagogía Profesional; Diploma Superior en Diseño de Proyectos; Diploma Superior en Sistemas de Educación Superior Modular Basados en Créditos Acumulables y Transferibles; Licenciada en Secretariado Ejecutivo; Universidad Estatal de Bolívar; Ecuador; rcedeno@ueb.edu.ec; (iD https://orcid.org/0000-0003-4997-356X

3. Magister en Gerencia Educativa; Diploma Superior en Gestión y Planificación Educativa; Ingeniero Comercial; Licenciado en Administración de Empresas; Universidad Estatal de Bolívar; Ecuador; mescobar@ueb.edu.ec; (iD https://orcid. org/0000-0002-7843-0145

4. Master Universitario en Modelización y Física de Sistemas Complejos; Magister en Gerencia Empresarial, MBA, mención Gestión de Proyectos; Diplomado Superior en Gestión de Proyectos; Físico; Universidad Estatal de Bolívar; Ecuador; smedina@ueb.edu.ec; (D) https://orcid.org/0000-0003-4494-0814

\section{CORRESPONDENCIA \\ Edelmira Lila Guevara Íñiguez \\ eguevara@ueb.edu.ec \\ Bolívar; Ecuador}




\section{RESUMEN}

En educación se debe utilizar la tecnología con todos sus avances y de la manera más eficaz. El considerar la tecnología como una creación humana permite aprovecharla para manejarla más fácilmente, para comprender el valor que tiene en los contextos educativos, por ejemplo como apoyo a la mediación que reclama el proceso de enseñanza y de aprendizaje en cualquiera de los niveles educativos y dentro de modelos formales y no formales. El ámbito educativo y, en consecuencia, el rol del docente, no ha podido resistirse a su influencia. La adecuación tanto del contenido como de las metodologías de enseñanza se ha convertido en un reto necesario, e incluso urgente, en un contexto social en cambio constante, en el que los jóvenes, rodeados de pantallas desde su nacimiento, han adquirido unos rasgos diferenciados a los de cualquier generación anterior. El presente artículo describe y compara diferentes literaturas sobre el rol del docente en la educación virtual. Para ello, se recolecta esta información de diferentes fuentes bibliográficas adquiridas de bases de datos (SCOPUS, PubMed, Biblioteca Cochrane, Google Scholar) valorando la calidad y veracidad de la información recopilada, así como la actualidad del contenido. En líneas generales, podemos concluir que sea en el entorno que sea, es decir, virtual o presencial, el rol del docente debe ser el mismo, el de ser un facilitador y transmisor de la información de una manera adecuada y coherente, que el alumno pueda captar dicha información y de esta manera generar el aprendizaje necesario. En este contexto en relación a la educación virtual el docente debe estar capacitado tecnológicamente para manejar las diferentes herramientas de las tecnologías de la información y comunicación para poder transmitir sus enseñanzas de una manera que genere la atención del alumno.

Palabras clave: Virtual, contenido, docente, aula, información.

\section{ABSTRACT}

In education, technology should be used with all its advances and in the most efficient way. Considering technology as a human creation allows us to take advantage of it to handle it more easily, to understand the value it has in educational contexts, for example as support for the mediation that demands the teaching and learning process at any of the educational levels and within of formal and non-formal models. The educational environment and, consequently, the role of the teacher, has not been able to resist its influence. The adequacy of both the content and the teaching methodologies has become a necessary, and even urgent, challenge in a constantly changing social context, in which young people, surrounded by screens since their birth, have acquired differentiated features. Those of any previous generation. This article describes and compares different literatures on the role of the teacher in virtual education. To do this, this information is collected from different bibliographic sources acquired from databases (SCOPUS, PubMed, Cochrane Library, Google Scholar), assessing the quality and veracity of the information collected, as well as the timeliness of the content. In general terms, we can conclude that it is in the environment that is, that is, virtual or face-to-face, the role of the teacher must be the same, that of being a facilitator and transmitter of information in an adequate and coherent way, that the student can capture this information and in this way generate the necessary learning. In this context, in relation to virtual education, the teacher must be technologically trained to handle the different tools of information and communication technologies in order to transmit their teachings in a way that generates the student's attention.

Keywords: Virtual, content, teacher, classroom, information.

\section{RESUMO}

Na educação, a tecnologia deve ser utilizada com todos os seus avanços e da maneira mais eficiente possível. Considerar a tecnologia como uma criação humana nos permite aproveitá-la para manuseá-la mais facilmente, para compreender o valor que ela tem em contextos educacionais, por exemplo, como suporte para a mediação que exige o processo de ensino e aprendizagem em qualquer um dos níveis educacionais e dentro de modelos formais e não formais. O ambiente educacional e, conseqüentemente, o papel do professor, não tem sido capaz de resistir a sua influência. A adequação tanto do conteúdo quanto das metodologias de ensino tornou-se um desafio necessário, e até urgente, em um contexto social em constante mudança, no qual os jovens, rodeados de telas desde seu nascimento, adquiriram características diferenciadas. As de qualquer geração anterior. Este artigo descreve e compara diferentes literaturas sobre o papel do professor na educação virtual. Para isso, estas informações são coletadas de diferentes fontes bibliográficas adquiridas de bancos de dados (SCOPUS, PubMed, Cochrane Library, Google Scholar), avaliando a qualidade e a veracidade das informações coletadas, bem como a atualidade do conteúdo. Em termos gerais, podemos concluir que é no ambiente, ou seja, virtual ou presencial, que o papel do professor deve ser o mesmo, o de ser um facilitador e transmissor de informações de forma adequada e coerente, que o estudante pode captar estas informações e desta forma gerar o aprendizado necessário. Neste contexto, em relação à educação virtual, o professor deve ser tecnologicamente treinado para lidar com as diferentes ferramentas das tecnologias de informação e comunicação, a fim de transmitir seus ensinamentos de forma a gerar a atenção do aluno.

Palavras-chave: Virtual, conteúdo, professor, sala de aula, informação. 


\section{Introducción}

En educación se debe utilizar la tecnología con todos sus avances y de la manera más eficaz. El considerar la tecnología como una creación humana permite aprovecharla para manejarla más fácilmente, para comprender el valor que tiene en los contextos educativos, por ejemplo como apoyo a la mediación que reclama el proceso de enseñanza y de aprendizaje en cualquiera de los niveles educativos y dentro de modelos formales y no formales (Tellería, 2004, pág. 210).

La incorporación de las TIC en la vida y la sociedad, demanda nuevos modelos de enseñanza-aprendizaje. Este modelo se centra en el alumno, el cual se convierte en el responsable de su propio proceso de aprendizaje; pasando de ser un ente pasivo a un actor con un rol muy activo e importante. En este caso el papel del profesor debe buscar y utilizar la metodología y emplear los recursos tecnológicos adecuados, para enriquecer y hacer eficaz el proceso de aprendizaje. Lo anteriormente expuesto implica el desarrollo del aprendizaje colaborativo y el individual, debe además desarrollar habilidades, aptitudes y valores de una manera programada, donde se evalúe su logro; utilizando una variedad de técnicas didácticas.
De este modo supera el rol tradicional de transmisor de información, y se convierte en creador de oportunidades de aprendizaje (Noris \& Aguilera, 2018, pág. 48).

La explosión informativa desencadenada por las TIC ha generado nuevas habilidades de acceso, evaluación y organización de la información en entornos digitales. Tanto los docentes como los discentes tienen que ser capaces de procesar y organizar la información, además de adecuarla y transformarla para crear nuevo conocimiento y usarlo como fuente de nuevas ideas. Todos requieren tener la capacidad de comunicar, intercambiar, criticar y presentar información e ideas con el uso de las TIC para favorecer la participación y contribución positiva en la cultura digital (Méndez \& Aguilar, 2013, pág. 110).

Cuando hablamos del enfoque de transmisión de Conocimientos adquiere gran importancia el material educativo que se convierte en la herramienta para transmitir contenidos, entonces vemos que lo tradicional no se refiere al tipo de materiales que se usan, que pueden ser libros o lo que usamos ahora, contenido multimedia, presentaciones on line, blogs, post, lo que es tradicional es el enfoque que se le da a la práctica pedagógica (Angarita Casas, 2015, pág. 9).

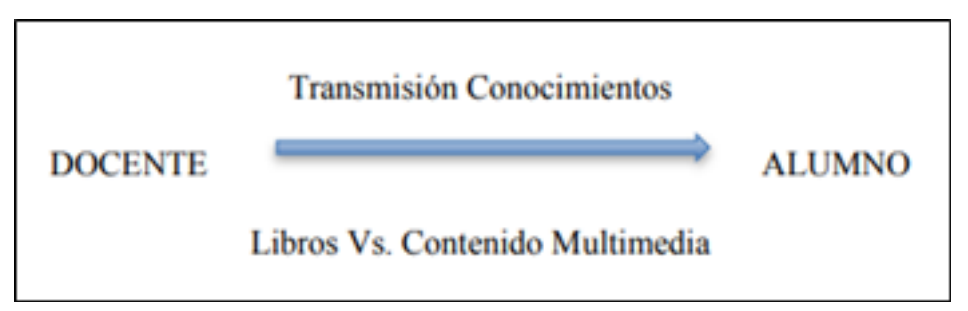

Imagen 1. Enfoque de transmisión de conocimientos

Fuente: (Angarita Casas, 2015) 
El ámbito educativo y, en consecuencia, el rol del docente, no ha podido resistirse a su influencia. La adecuación tanto del contenido como de las metodologías de enseñanza se ha convertido en un reto necesario, e incluso urgente, en un contexto social en cambio constante, en el que los jóvenes, rodeados de pantallas desde su nacimiento, han adquirido unos rasgos diferenciados a los de cualquier generación anterior (Blanco \& Amigo, 2016, pág. 104).

Para comprender el alcance de la buena práctica docente, Rodríguez \& Estay-Niculcar (2016) citando a Rodríguez (2012) lista una serie de indicadores que resaltan su potencial: primero, las buenas prácticas permiten la generación de un aprendizaje significativo aplicable a la vida diaria. Segundo, involucran a los alumnos en las actividades de aprendizaje ya que trabajar con buenas prácticas requiere una mayor cantidad de operaciones mentales, trabajo colaborativo y estrategias de autoaprendizaje autónomas. Tercero, las buenas prácticas promueven el pensamiento divergente. Cuarto, en las buenas prácticas intervienen diferentes campos de conocimiento (interdisciplinariedad y transversalidad), de igual forma establecen interconexiones entre los actores del proceso educativo y también entre grupos de trabajo e instituciones educativas. Finalmente, las buenas prácticas favorecen el uso de las TIC y exigen una evaluación continua (pág. 213).

Por lo tanto, habrá que tener en mente que en la educación virtual el docente debe ser considerado como un elemento central para lograr el adecuado desarrollo del proceso de aprendizaje. Por ello será conveniente que él mismo participe en su formación permanente relacionada con los principios teóricos de la educación virtual, las funciones de la tutoría, la asesoría, y que adquiera y enriquezca sus habilidades en cuanto al manejo de tecnologías orientadas a la educación de esta naturaleza (Garduño Vera, 2007, pág. 159).

\section{Metodología}

El presente artículo describe y compara diferentes literaturas sobre el rol del docente en la educación virtual. Para ello, se recolecta esta información de diferentes fuentes bibliográficas adquiridas de bases de datos (SCOPUS, PubMed, Biblioteca Cochrane, Google Scholar) valorando la calidad y veracidad de la información recopilada, así como la actualidad del contenido.

\section{Resultados}

El rol del docente no es algo que se pueda precisar desde un solo punto de vista, hay que revisar varios aspectos que inciden a la hora de definir y delimitar sus características y competencias. De acuerdo con las lecturas es importante mirar tres aspectos desde donde comienza a tomar forma el rol virtual, el primero está dado por la sociedad que inminentemente determina que espera la comunidad del docente, también se define a partir de la normatividad, estamos en un estado de derecho, donde el actuar de las instituciones educativas, de directivos y docentes se define en gran parte por los decretos y gobernantes de turno, por eso la importancia de revisar esta documentación. Y por último, está la mirada a partir del mismo docente, de lo que él propone como profesional, este apartado se denomina Formación profesional y se genera a partir de la importancia de dar una revisión a los programas que se ofertan para los docentes y lo que proponen las instituciones educativas en términos de formación y capacitación (Angarita Casas, 2015).

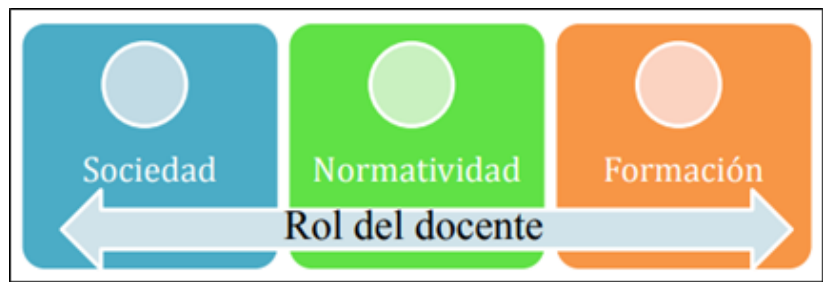

Imagen 2. Rol del Docente

Fuente: (Angarita Casas, 2015) 
Analizando el papel de los docentes virtuales, estos tienen como reto el desarrollar nuevas habilidades que les permitan asegurar un uso eficiente de las herramientas informáticas, y mantener despierto en sus alumnos el interés y la curiosidad por el aprendizaje mediante el uso de estas plataformas. Como lo manifiestan Zambrano y García (2010) citado por Céspedes (2018), el docente-tutor debe como primera medida manejar adecuadamente las tecnologías Web 2.0, desarrollar estrategias de enseñanza apropiadas para este entorno educativo, debe además ser un guía, motivador, facilitador y supervisor de las estrategias que utilicen sus alumnos, debe estar atento a las dificultades que puedan presentar los mismos y tener la capacidad para planear las actividades y evaluarlas. Todo esto con el fin de desarrollar eficientemente todo el proceso de enseñanza-aprendizaje.

En un modelo de enseñanza-aprendizaje centrado en el aprendizaje del alumno, el papel del profesor es ante todo, facilitar el aprendizaje, es decir de diseñar situaciones para que los alumnos aprendan y orientarlos en la realización de las actividades que dichas situaciones suponen, de tal modo que puedan alcanzar los objetivos previstos. De este modo supera el rol tradicional de transmisor de información, y se convierte en creador de oportunidades de aprendizaje (Salinas, 2011).

En este diseño habrá que prestar especial atención a algunos aspectos, tales como:

- Promover procesos de participación, interacción y colaboración, de tal forma que los alumnos puedan apropiarse del conocimiento en forma activa e interactiva.

- Ejercer una tutoría constante del proceso de aprendizaje, es decir actuar como guía durante el desarrollo de dicho proceso, orientando al alumno en la ejecución de las tareas previstas. Esto se relaciona también con la práctica de la evaluación continua.

- Actuar como animador y moderador de la comunicación intragrupal: planificar instancias de interacción con el propio docente y con los pares, que podrán ser sincrónicas o asincrónicas; gestar un clima relacional positivo en el grupo, capaz de estimular la participación y la interacción comunicativa; motivar la participación de quienes intervengan poco, diluir eventuales conflictos dentro del grupo, establecer normas para regular los aspectos formales del intercambio (estilo y tono de los mensajes, extensión), etc (Salinas, 2011).

En este contexto Ryan y otros (2000) citado por Noris \& Aguilera 2018) indican que, El rol central del docente es el de actuar como mediador o intermediario entre los contenidos y la actividad constructivista que despliegan los alumnos para asimilarlos. Los roles y responsabilidades del moderador en la actividad docente en línea se pueden agrupar en cuatro categorías: pedagógica, social, administrativa y técnica según; En lo pedagógico: El tutor es un facilitador que contribuye con el conocimiento especializado, focaliza la discusión en puntos críticos, hace las preguntas y responde a las contribuciones de los participantes, le da coherencia a la discusión, sintetiza los puntos, destacando los temas emergentes.

- En lo social: necesita habilidades para crear una atmósfera de colaboración que permita generar una comunidad de aprendizaje.

- En el aspecto técnico: Debe garantizar que los participantes se sientan cómodos con el software y si es necesario apoyarlos.

- En lo administrativo: Conocer el software para poder generar subconferencias, grupos de trabajos y poder mover o borrar mensajes de la conferencia.

En relación al rol del tutor se plantean nuevas competencias y habilidades que estos

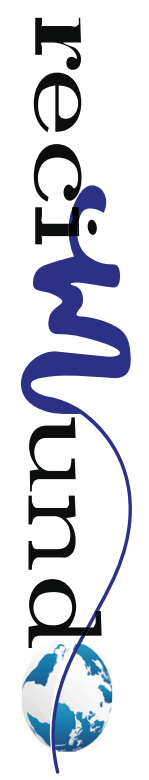


profesionales deben dominar para utilizar el potencial pedagógico de estos espacios. El docente debe estar preparado para generar un diálogo efectivo con los participantes y entre los participantes, que favorezca el aprendizaje activo, la construcción de conocimiento cooperativo y/o colaborativo. Este cambio de rol demanda el replanteamiento en las actitudes, en los modos y formas de actuación e interacción y sobre todo implica la toma de conciencia de su papel como mediador a través de procesos intencionales de intervención pedagógica previa, sincrónica o asincrónica.

Barker (2002) citado por Noris \& Aguilera (2018) establece un conjunto de competencias que debería poseer un tutor online al llevar a cabo un proceso formativo a través de Internet, como son:

Competencias pedagógicas:

- Profundizar / investigar temas.

- Estructurar el conocimiento.

- Diseño de tareas individualizadas para el autoaprendizaje.

- Diseño de actividades de trabajo en grupo.

- Formular estrategias de valoración.

- Guiar, aconsejar y proporcionar feedback.

Competencias técnicas:

- Utilizar adecuadamente el correo electrónico.

- Saber dirigir y participar en comunicaciones asincrónicas.

- Diseñar, crear y controlar las salas de chat sincrónicas

- Dominar y utilizar procesadores de texto, hojas de cálculo y bases de datos.

- Utilizar herramientas de creación de páginas web.

- Usar el software con propósitos determinados.

Competencias organizativas:

- Seleccionar y organizar a los estudian- tes para realizar actividades grupales.

- Establecer estructuras en la comunicación online con una determinada lógica.

- Organizar a los estudiantes teniendo en cuenta sus datos geográficos.

- Recopilar y archivar los trabajos de los estudiantes para su posterior valoración.

- Organizar las tareas administrativas.

Son muchos los docentes que, por iniciativa propia, han decidido renovarse con el objetivo de seguir preparando al alumnado para el mundo que les toca; sin embargo, son también muchas las reacciones contrarias que han provocado que exista un rechazo ante estos cambios motivados por la tecnologización de la vida y las escuelas. Existe un cierto temor ante el uso de las TIC e Internet y sus consecuencias. Además, los medios de comunicación no han contribuido a proyectar las ventajas de la red, por lo que, de entrada, parece haberse instalado una sensación de inseguridad que ha repercutido en el ámbito educativo formal (Blanco \& Amigo, 2016).

En ningún caso el docente debe convertirse en un controlador o policía de lo que hacen sus estudiantes en el aula. Su función es coordinar y facilitar el aprendizaje y la mejora de la calidad de vida del alumnado. Si bien es cierto que el aprendizaje debe ser experiencial y activo por parte de este, en todo momento es preciso el complemento de un docente que le acompañe en su proceso de aprendizaje. El conocimiento está en la red y es abundante, pero precisamente esto es lo que hace necesario un buen número de tareas que debe cumplir todo docente: detectar lo realmente importante, guiar los procesos de búsqueda, analizar la información encontrada, seleccionar la que realmente se necesita, interpretar los datos, sintetizar el contenido y difundirlo son algunas de las tantas tareas que el profesor debe guiar (Blanco \& Amigo, 2016).

En definitiva, el docente de la Era Digital debe mantener una actitud de indagación 
permanente, fomentar el aprendizaje de competencias (generar entornos de aprendizaje), mantener una continuidad del trabajo individual al trabajo en equipo (apostar por proyectos educativos integrados) y favorecer el desarrollo de un espíritu ético. La tecnología y la información por sí solas no guían ni ayudan ni aconsejan al alumnado; por ello, la labor del docente en la educación digital es hoy más importante que nunca (Blanco \& Amigo, 2016).

\section{Conclusiones}

En líneas generales, podemos concluir que sea en el entorno que sea, es decir, virtual o presencial, el rol del docente debe ser el mismo, el de ser un facilitador y transmisor de la información de una manera adecuada y coherente, que el alumno pueda captar dicha información y de esta manera generar el aprendizaje necesario. En este contexto en relación a la educación virtual el docente debe estar capacitado tecnológicamente para manejar las diferentes herramientas de las tecnologías de la información y comunicación para poder transmitir sus enseñanzas de una manera que genere la atención del alumno. En el contexto actual en donde la humanidad intenta retomar la normalidad debido a la pandemia del Covid-19, se puso como nunca antes a prueba la capacidad de los profesores y alumnos en los diferentes ámbitos educativos de utilizar el ámbito virtual para generar los procesos de enseñanza, a muchos estamos seguros les ocasiono dificultades, a otros solo pulir algunas cosas y otros de esta generación que ya dominan las redes y las Tics fue tramite mas.

\section{Bibliografía}

Angarita Casas, K. E. (2015). Rol del docente en la Educación Virtual.

Blanco, A. V., \& Amigo, J. (2016). El rol del docente en la era digital. Revista interuniversitaria de formación del profesorado: RIFOP, 86, 103-114.

Céspedes, P. A. (2018). La calidad de la educación universitaria, educación virtual y rol del docente-tutor. CITED Journal, 1(1), 10-10.

Garduño Vera, R. (2007). Caracterización del docente en la educación virtual: consideraciones para la Bibliotecología. Investigación bibliotecológica, 21(43), 157-183.

Méndez, M. D., \& Aguilar, G. (2013). Quehacer docente, TIC y educación virtual oa distancia. Apertura, 5(2), 108-123.

Noris, L. M., \& Aguilera, Y. (2018). Papel del docente en los Entornos Virtuales de Aprendizaje. Open Journal Systems en Revista: REVISTA DE ENTRENAMIENTO, 1(2), 47-62.

Rodríguez, R. D., \& Estay-Niculcar, C. (2016). Formación en buenas prácticas docentes para la educación virtual. RIED. Revista Iberoamericana de Educación a Distancia, 19(1), 209-232.

Salinas, M. I. (2011). Entornos virtuales de aprendizaje en la escuela: tipos, modelo didáctico y rol del docente. Universidad Católica de Argentina, $1-12$.

Tellería, M. B. (2004). Educación y nuevas tecnologías. Educación a Distancia y Educación Virtual. Revista de Teoría y Didáctica de las Ciencias Sociales, 9, 209-222.

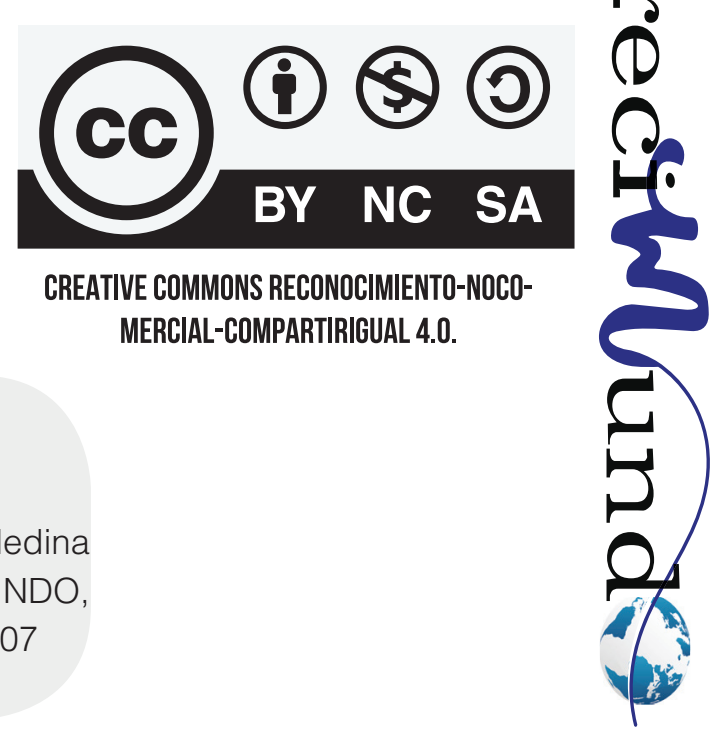

\section{CitAR este ARTICULO:}

Guevara Íñiguez, E. L., Cedeño Álvarez, R. C., Escobar Gortaire, M. E., \& Medina Velasco, S. R. (2021). El rol del docente en la educación virtual. RECIMUNDO 5(3), 101-107. https://doi.org/10.26820/recimundo/5.(2).julio.2021.101-107 